\title{
Evaluación de programas educativos en instituciones musicales El caso Adoptar un Músico
}

Evaluation of educational programmes in music institutions. The case of "Adopt a Player"

\author{
Manuel Cañas Escudero \\ manuelce@ugr.es \\ José A. Rodríguez-Quiles \\ kiles@ugr.es \\ Facultad de Ciencias de la Educación \\ Universidad de Granad \\ Campus de Cartuja, $\mathrm{s} / \mathrm{n}$ \\ 18170 Granada
}

doi: 10.7203/LEEME.39.9926

\section{Resumen}

Orquestas, Auditorios y Espacios Escénicos (las instituciones musicales más representativas de la expresión pública de la música) vienen desarrollando desde hace años una amplia variedad de acciones educativas, con especial atención hacia el público joven, y con incidencia notable en colectivos muy numerosos. En ocasiones, estas acciones se configuran como programas educativos estables que generan espacios de experiencia y aprendizaje, tanto en la educación no formal como en la educación formal de carácter reglado. Este trabajo aborda la evaluación de uno de estos programas: Adoptar un Músico, de la Orquesta y Coro Nacionales de España. El objetivo fundamental es identificar y analizar los efectos que produce en quienes participan de los mismos y las implicaciones que se derivan de ello. El planteamiento metodológico es mixto, cualitativo y cuantitativo, incluyendo observaciones, entrevistas, cuestionarios y revisión documental. Aquí se ofrece un panorama general de la investigación, resultados y conclusiones. Los resultados evidencian efectos significativos en el proceso educativo y en factores del aprendizaje, cognitivos, conductuales, emocionales y sociales, ligados a una práctica musical compartida, activa y creativa.

Palabras clave: educación musical; investigación educativa; evaluación de programas educativos; instituciones musicales.

\section{Abstract}

For years, orchestras, concert halls and theaters (the most representative musical institutions of public expression of music), have been developing a wide variety of educational activities, with special emphasis on young people, and a very significant impact on other groups of people. Sometimes, these actions are configured as stable educational programs that create opportunities for experience and learning, both in non-formal education and formal education of a regulated nature. This paper addresses the evaluation of the "Adopt a Player" program of the National Orchestra and Choir of Spain. The main objective is to identify and analyze the effects this program has on the participants and the implications arising from those outcomes. The methodological approach is mixed, qualitative and quantitative, including observations, interviews, questionnaires, and document review. Here, an overview of the research results and conclusions is offered. The results show significant effects on the educational process and learning factors, cognitive, behavioral, emotional and social, linked to a shared, active, and creative musical practice.

Keywords: music education; educational research; evaluation of educational programs; musical institutions.

\section{Introducción}

Desde hace más de veinte años, las Instituciones Musicales en España (orquestas, auditorios y espacios escénicos) vienen desarrollando diversas actividades educativas con incidencia socioeducativa en sus ámbitos de influencia. Estas actividades, como los conciertos didácticos, permiten "acceder a la música en las mejores condiciones posibles de interpretación y producción. Conectan a niñas y niños, 
jóvenes y familias con la experiencia de la música en vivo, utilizando procedimientos didácticos adecuados para cada auditorio y circunstancia" (Cañas, 2008, p.12). Se configuran dentro de modelos educativos de aprendizaje no formal, si bien en su desarrollo alcanzan espacios de contacto (que pueden llegar a ser de integración) con los modelos formales de carácter reglado. Son actividades que centran su atención en aprendizajes prácticos, adquiridos de los propios participantes (intérpretes, docentes, alumnado) y de otros agentes educativos que puedan intervenir, expertos o no en el ámbito musical y derivados de experiencias y reflexiones compartidas.

Conviene precisar que los modelos formales se caracterizan por un contexto organizado explícitamente para el aprendizaje intencional, con un plan de estudios reglado y estructurado que tiene por objeto la adquisición de competencias específicas y que permite un reconocimiento y acreditación normalizados. En cambio, los modelos no formales se refieren a una actividad educativa organizada fuera de un marco normativo, destinada a colectivos definidos, aunque con carácter abierto, y adaptada a las necesidades del grupo. Con un plan menos estructurado, tienen por objeto la adquisición de competencias diversas, más transversales, y propicia un aprendizaje tanto intencional como accidental.

Aun teniendo algunas zonas de interés común con nuestra investigación, en este estudio no se considerarán los programas de música comunitarios, que, sin duda, son susceptibles de un tratamiento propio y extenso por su relevante interés. Entre otras razones, entendemos que "las propuestas de música comunitaria son espacios de aprendizaje de la música, pero ubicados al margen del sistema educativo formal" (Cabedo y Díaz, 2016, p.5). De igual manera lo enfoca Higgins cuando afirma que "los músicos que trabajan de este modo generalmente lo hacen fuera de ambientes formales de enseñanza y aprendizaje, facilitando experiencias para la creación musical grupal en entornos que no tienen planes curriculares establecidos" (Higgins, 2013, p.54).

Un número muy amplio de docentes del sistema educativo reglado, principalmente de música, participan en las citadas actividades, de forma habitual y en diversos grados. La dimensión cuantitativa de este fenómeno es notable, si atendemos a los siguientes datos ${ }^{1}$ :

- ROCE, Red de Organizadores de Conciertos Educativos, es una asociación que integra a diversas instituciones (orquestas, auditorios, fundaciones...) que desarrollan programas educativos de música en España. En la temporada 2012, sus instituciones asociadas celebraron un total de 1.355 conciertos de carácter educativo, en 206 producciones diferentes, con una asistencia de 577.982 personas. Además, impulsaron 93 actividades paralelas, transversales o complementarias, de las que se realizaron un total de 855 sesiones, a las que asistieron 26.684 personas (ROCE, 2016).

- Lacord, Plataforma de Concerts Educatius de Catalunya, es una entidad integrada por 22 instituciones, equipamientos y entidades públicas y privadas que se dedican profesionalmente a la creación, producción y difusión de conciertos educativos. Durante la temporada 2011 desarrollaron en esa Comunidad 2.254 conciertos y otras

\footnotetext{
${ }^{1}$ A fecha de redacción del presente texto, diciembre 2016, los datos aportados son los últimos publicados por cada uno de los organismos citados: ROCE, 06.07.2013. Lacord, 26.10.2011. ABO, 09.01.2014. AFO, 05.10.2015. 
1.940 actividades educativas, en 221 producciones diferentes, con un total de 763.626 asistentes (Lacord, 2011, p.3).

A modo de referencia para una perspectiva comparada, según la Association of British Orchestras, durante la temporada 2013 se realizaron en el Reino Unido 2.241 conciertos educativos con 326.208 asistentes, y otras 8.387 actividades educativas en las que participaron 335.579 personas(ABO, 2014, p.3).En el caso de la Association Française des Orchestres, las actividades educativas desarrolladas en 2013 por 13 de sus orquestas asociadas registraron una participación de 226.000 espectadores, un $20 \%$ de la asistencia total(AFO, 2014, p.7).

Como afirma Korn (2000) en su referencia a conciertos y actividades educativas, "many (orchestras) have developed over time, without strategic thought or planning" (p.60). No obstante, en ocasiones, las acciones desarrolladas sí se configuran dentro de programas educativos estables en el tiempo, "programs contain meaningful learning, opportunities for families, and build capacity in four critical areas: professional development of teachers and administrators, artist training, curriculum development, and assessment" (p.68). Algunas Instituciones Musicales organizan diferentes programas educativos, conformando un conjunto de acciones que desarrollan eficazmente los objetivos de un proyecto educativo global y singularizado sobre una filosofía propia. Este tipo de proyectos aborda "el encuentro entre música y jóvenes desde una perspectiva plenamente educadora" (Cañas, 2008, p.14), "al servicio de la educación musical de nuestro entorno y atendiendo a su constante evolución" (Malagarriga, 2010, p.147).

Este trabajo aborda la evaluación de uno de estos programas: Adoptar un Músico, de la Orquesta y Coro Nacionales de España, con el objetivo de identificar y analizar los efectos que produce en quienes participan de los mismos y las implicaciones que se derivan de ello. Aquí se ofrece un panorama general de la investigación, resultados y conclusiones.

\section{Estado de la cuestión}

La evaluación de programas educativos en este campo no es una práctica extendida en nuestro país ni se ha normalizado como un aspecto básico dentro de la planificación de cualquiera de los programas que se diseñan. La práctica más frecuente en algunas Instituciones Musicales ha sido la aplicación de cuestionarios para conocer el grado de satisfacción de quienes participan en una actividad concreta, principalmente profesorado y alumnado. Para ello, se formula un número limitado de preguntas con el objeto de obtener datos cuantitativos sobre algunas variables de interés inicial: perfil, hábitos, valoración... Con el tiempo, este procedimiento tiende a caer en un hábito rutinario, sin referencia en un proceso evaluativo global y con escaso valor significativo.

Es reducida la producción de trabajos con referencia específica a este ámbito de actividad ${ }^{2}$. Por lo general, se estructuran como estudios, informes, actas... procedentes no de las propias Instituciones

\footnotetext{
${ }^{2}$ Por motivos de espacio sólo se mencionarán aquí las publicaciones más relevantes para el objeto específico de nuestro estudio, es decir, la evaluación de estos programas. No se incluyen, por tanto, referencias a otras publicaciones que puedan aludir a dichos programas desde perspectivas diferentes.

THE CONTENT OF THIS ARTICLE IS THE SOLE RESPONSIBILITY OF THE AUTHORS. THE REVISTA ELECTRÓNICA DE LEEME AND UNIVERSITAT DE VALĖNCIA ARE NOT LIABLE FOR ANY LEGAL ACTIONS THAT MAY ARISE INVOLVING THE ARTICLE'S CONTENT. REVISTA ELECTRÓNICA DE LEEME -LISTA ELECTRÓNICA EUROPEA DE MÚSICA EN LA EDUCACIÓN-HTTP://MUSICA.REDIRIS.ES.ISSN: 15759563 EDITORES. UNIVERSIDAD DE VALENCIA Y JESUS T JADA GMENEZ. VISIBILIDAD DE ESTA REVSTA. EBSCO, CINDOC (CESIC), CITEFACTOR, COPAC, DIALNLT, DICE (CSIC), DOAJ, EREVISTAS ESTA REVISTA ESTA PUBLICADA CON EL APOYO INSTITUCIONAL DE REDIRIS-CONSEJO SUPERIOR DE INVESTIGACIONES CIENTIFICAS Y ES DE ACCESO LIBRE.
} 
Musicales sino de ámbitos académicos o publicaciones especializadas. Podemos observar los textos disponibles atendiendo al colectivo de interés sobre el que se incide. Respecto al alumnado, el de Moreiras (2005, p.102), relativo a un concierto de la Orquesta Sinfónica de Galicia para escolares de educación secundaria, y el de Swol (2005, p.47), analizando los conciertos didácticos organizados por la Orquesta de Córdoba. Respecto al profesorado, el de Neuman (2003), centrado en los conciertos didácticos de la Orquesta Ciudad de Granada, y el de Ortega (2012), con referencias al programa Adoptar un músico.

En un tercer grupo podríamos situar los que recaban datos tanto del profesorado como del alumnado: Lukas, Santiago y Lizasoain (2005) evaluaron los programas del Departamento Municipal de Educación de Vitoria, entre ellos, el Programa de Expresión Musical, y Ferrada (2008) se interesó porlos ensayos de la Orquesta Sinfónica de Barcelona. En una óptica distinta, la de público familiar, el de Ramírez (2006), que investigó el público de Ópera en Familia en el Teatro Real de Madrid, y Blázquez y Bidegain (2016), sobre los conciertos familiares y participativos del Auditorio de Barcelona.

En este contexto, cabe apuntar que Vinent y Gustems (2014) aprecian como una debilidad "la falta de un estudio de impacto y del seguimiento del trabajo que realizan los profesores en la escuela impide establecer la verdadera pertinencia de los conciertos didácticos dentro del proceso de enseñanza aprendizaje" (p.48).El balance general constata que, lejos de activarse procesos regulares de evaluación de los programas educativos que desarrollan las Instituciones Musicales, las experiencias se han limitado a prácticas puntuales y de alcance limitado. En síntesis, hemos de considerar que este tipo de programas son susceptibles (y, objetivamente, demandan) un tratamiento evaluativo global, tanto de los procesos presentes en ellos como de los impactos generados. Todo ello, a fin de posibilitar los cambios necesarios: un proyecto educativo orienta un desarrollo de calidad en base a su evaluación.

\section{Objetivos}

Se trata de averiguar y comprender qué sucede en el programa educativo que se investiga, así como de interpretar su significado y efectos. Se sitúa, por tanto, en sintonía con las características de la investigación cualitativa que señala Bresler (2006, p.65): holística, empírica, descriptiva, interpretativa y empática. En el marco de un contexto particular, quiere penetrar en su interior y comprender. En términos de Angrosino (2012), "captar la orografía del paisaje" y "obtener la perspectiva de las propias personas sobre los problemas" (p.41). Por tanto, es un enfoque que no está dirigido hacia un determinado tipo de estándares preestablecidos, sino que se centra en las personas que intervienen y en sus propias experiencias como ejes sobre los que pivotar. Así, el planteamiento de la investigación está orientado por los modelos de evaluación comprensiva de Stake (2006), en el de evaluación democrática de MacDonald (1976), entendida como servicio de información a la comunidad, y en el de evaluación personalizada de Kushner (2002).

Al mismo tiempo, la investigación coincide con la definición que hace Kelly (2008, p.303) en cuanto que busca la producción de un conocimiento que contribuya a una mayor comprensión de los efectos; también, hacia la posibilidad de mejora en el diseño y desarrollo de dichos programas. Además, 
atiende a lo propugnado por Elliott y Kushner (2007, p.332) acerca del diseño de la evaluación, que debería estar orientada a proporcionar información sobre los propósitos y la práctica educativa, incluyendo cuestiones de currículo, filosofía e implicación social. Los objetivos generales que se plantean son:

- Explorar, descubrir y comprender los procesos y dinámicas presentes en el programa.

- Obtener evidencia empírica sobre sus efectos en el tejido social y educativo.

- Analizar y valorar el impacto del programa en quienes intervienen.

- Identificar sus fortalezas, debilidades y posibles cambios.

Adicionalmente, el trabajo desea contribuir a la normalización en las Instituciones Musicales de la práctica de este tipo de evaluación, integrándola en el propio diseño de sus programas educativos. Todo ello, en el horizonte de configurar Instituciones Musicales que "aprenden" y "educan".

\section{Método}

El proyecto se configura como una investigación evaluativa con estudio de caso, de metodología mixta y diseño convergente, fundamentalmente cualitativa e integrando también técnicas cuantitativas complementarias. A continuación, se describe el marco metodológico, la selección del caso, el contexto y el proceso de la investigación, incluyendo observaciones, entrevistas y cuestionario.

En el campo de la educación musical, este planteamiento como estudio de caso está en coincidencia con lo defendido por Bresler (2006, p.65) cuando afirma la necesidad de comprensiones experienciales de situaciones particulares y Kushner (1998, pp. 245-246), quien reflexiona sobre la necesidad de una perspectiva más amplia y compleja en la evaluación educativa, abogando por "fundar la evaluación de la enseñanza musical en el empleo de estudios de casos" al tiempo que alertaba de una falta de estudios que uniesen los sucesos del aula con los contextos. Precisamente el contexto es un referente básico para autores como Yin (2009, p.18) y Simons (2011, p.42). Por otro lado, recordamos los planteamientos de Stake (1998, pp.17-19) y Flinders y Richardson (2002, p.1170) respecto a que la investigación con estudio de casos no es una investigación de muestras con voluntad generalizadora.

El interés del carácter mixto de la metodología empleada está respaldado por diversos autores como Johnson, Onwuegbuzie, Burke, Creswell, Planco y Klassen. Así, Johnson y Onwuegbuzie (2004, p.17) definieron los diseños mixtos como la clase de investigación donde se mezcla o combinan técnicas, métodos, enfoques, conceptos o lenguaje de investigación cuantitativos y cualitativos en un solo estudio. Por su parte, Burke (2004, p.21) establece que uno de los puntos fuertes de la investigación cualitativa y cuantitativa, utilizada en conjunto, es que producen un conocimiento más completo, necesario para informar a la teoría y la práctica. Burke (2007, p.127) reitera esta fortaleza en la metodología mixta, subrayando la recogida de todos los datos que puedan proporcionar información potencialmente relevante para el objetivo de la investigación.

Creswell (2008, p.526) define la metodología mixta como aquella en la que el investigador recoge y analiza datos, integra hallazgos y dibuja inferencias usando ambos enfoques o métodos, THE CONTENT OF THIS ARTICLE IS THE SOLE RESPONSIBILITY OF THE AUTHORS. THE REVISTA ELECTRÓNICA DE LEEME AND UNIVERSITAT DE VALĖNCIA ARE NOT LIABLE FOR ANY LEGAL ACTIONS THAT MAY ARISE INVOLVING THE ARTICLE'S CONTENT. REVISTA ELECTRÓNICA DE LEEME -LISTA ELECTRÓNICA EUROPEA DE MÚSICA EN LA EDUCACIÓN-HTTP://MUSICA.REDIRIS.ES.ISSN: 1575(CSIC), EBSCO PREMIER, ERIH+, GALE CENGAGE LEARNING, IN-RECS, IRESIE, LATINDEX, MIAR, OCLC WORLDCAT, RESH, REDIB, RILM CORE JOURNALS, SUDOC, ULRICHS, ZEITSCHRIFTDATENBANK, ESTA REVISTA ESTÁ PUBLICADA CON EL APOYO INSTITUCIONAL DE REDIRIS-CONSEJO SUPERIOR DE INVESTIGACIONES CIENTIFICAS Y ES DE ACCESO LIBRE. 
cualitativo y cuantitativo, en un solo estudio. Dentro de su tipología de prototipos de métodos mixtos, Creswell y Planco (2011, p.77) establecen el diseño convergente, basado en la recogida y análisis de datos cuantitativos y cualitativos en la misma fase de la investigación, combinando los dos conjuntos de resultados en una interpretación global. Así, la síntesis de resultados complementarios permite desarrollar una comprensión más completa del fenómeno. Klassen et al. (2012, p.379) también señalan el uso del diseño convergente cuando la intención es fusionar datos concurrentes, cuantitativos y cualitativos.

En esta investigación, el estudio de caso se ofrece como una metodología coherente con los objetivos ya señalados. Por su parte el carácter mixto de diseño convergente permite disponer de un mayor volumen y tipología de datos valiosos al tiempo que posibilita el acceso a una muestra de informantes más amplia.

\subsection{Selección del caso. El programa Adoptar un músico}

Para la finalidad de la investigación, la selección del caso no se formula mediante muestreo estadístico sino mediante muestreo intencional basado en determinados criterios, de acuerdo con autores como Goetz y LeCompte (1988, p.98) y Flick (2004, pp.80-81):

El principio básico del muestreo teórico es seleccionar casos o grupos de casos según criterios concretos acerca de su contenido en lugar de utilizar criterios metodológicos abstractos. El muestreo procede según la relevancia de los casos, en lugar de hacerlo según su representatividad. (...) es la forma genuina y típica de seleccionar el material en la investigación cualitativa.

También Díaz (2006, p.111), en la selección de la muestra, alude al muestreo no probabilístico, que se ajusta a criterios relacionados con las características de la investigación. La relación de Instituciones Musicales que operan en España con programas educativos es extensa. Una vez explorada la población en la práctica, y acotados los casos relevantes, se decidió focalizar la atención hacia Instituciones Musicales y programas que presentaban determinadas características (tabla 1.):

- Diferente tipología (Orquesta, Auditorio, Espacios Escénico).

- Desarrollo de programas de forma estable y continuada, preferentemente con un periodo de vigencia de al menos cinco años.

- Programas que implicasen algún formato de participación activa de sus protagonistas. 
Tabla 1. Selección de casos

\begin{tabular}{|c|c|c|}
\hline Institución Musical & Programa & Año de inicio \\
\hline L'Auditori & Cantania & 1989 \\
\hline Orquesta y Coro Nacionales de España & Adoptar un músico & 2005 \\
\hline Teatro Real & La Ópera, un vehículo de aprendizaje & 2007 \\
\hline Centro Nacional de Difusión Musical & Todos creamos & 2012 \\
\hline
\end{tabular}

De los casos citados, el presente texto ${ }^{3}$ se ocupa de la investigación realizada respecto a Adoptar un músico, un programa en el que concurren, además, dos aspectos singulares: la existencia de proyectos instrumentales y vocales, y la interacción músicos-aulas. Iniciado en los años 80 en el Reino Unido, se desarrolla desde 2004 en la OCNE, Orquesta y Coro Nacionales de España, con la colaboración del Departamento de Educación Artística del Centro Regional de Innovación y Formación de la Consejería de Educación, Juventud y Deporte de la Comunidad de Madrid, CRIF "Las Acacias".

En cada edición del programa, destinado a grupos de alumnado de Educación Primaria y Secundaria de centros participantes (de 4 a 6 centros de la Comunidad de Madrid), interviene el músico de la OCNE "adoptado" por cada centro, una persona experta en estos proyectos educativos (director musical, compositor...) y un asesor o asesora del CRIF, junto al profesorado de música y tutor correspondiente. El marco formal tiene estos objetivos:

a) Conseguir una escucha musical más activa y participativa, convirtiendo en creadores e intérpretes al profesorado y alumnado.

b) Acercar al alumnado a los elementos y estructuras de la música, haciéndoles partícipes en las fases de creación e interpretación.

c) Estimular la creatividad artística y el espíritu crítico de los participantes.

d) Entender y disfrutar la riqueza del patrimonio musical

e) Promover relaciones entre la OCNE, profesorado y alumnado, desmitificando la música sinfónico-coral y a sus protagonistas.

En la práctica, el objetivo final es que, en base a una obra musical programada por la OCNE, cada grupo llegue a componer su propia obra y la interprete en un concierto en el Auditorio Nacional. En una primera fase, se realizan sesiones de formación en el Auditorio Nacional de Música y en el CRIF con el profesorado y músicos implicados, en los que se trabaja sobre la metodología creativa que se utilizará, las técnicas compositivas, la instrumentación, y se planifica el trabajo. En la segunda fase, los músicos se desplazan a los centros educativos para trabajar en talleres el conocimiento y la recreación del material sonoro y, a continuación, en la creación sonora y coreográfica de la propia obra del grupo. Finalmente, después de meses de trabajo, cada programa implica ensayos previos y un concierto que se ofrece a toda la comunidad educativa y público en general. Hasta 2014 se habían desarrollado 33

\footnotetext{
${ }^{3}$ En breve se podrá consultar información adicional sobre la investigación en un texto más detallado y extenso.

THE CONTENT OF THIS ARTICLE IS THE SOLE RESPONSIBILITY OF THE AUTHORS. THE REVISTA ELECTRÓNICA DE LEEME AND UNIVERSITAT DE VALĖNCIA ARE NOT LIABLE FOR ANY LEGAL ACTIONS THAT MAY ARISE INVOLVING THE ARTICLE'S CONTENT. REVISTA ELECTRÓNICA DE LEEME -LISTA ELECTRÓNICA EUROPEA DE MÚSICA EN LA EDUCACIÓN-HTTP:/ / MUSICA.REDIRIS.ES.ISSN: 1575(CST) ESTA REVISTA ESTÁ PUBLICADA CON EL APOYO INSTITUCIONAL DE REDIRIS-CONSEJO SUPERIOR DE INVESTIGACIONES CIENTIFICAS Y ES DE ACCESO LIBRE.
} 
programas diferentes de Adoptar un músico, de carácter instrumental o vocal, en los que participaron 155 centros, 292 docentes y 4.252 alumnos.

\subsection{Contexto}

Como en otros periodos, el programa Adoptar un músico 2013 - 2014 se desarrolló en escenarios muy diversos e implicó a personas y colectivos que se hallan inmersos en dos culturas con rasgos propios muy acusados, específicos y diferenciados entre sí: el educativo de niveles básicos y el musical de máximo nivel.

Por un lado, 18 centros educativos que atienden a niveles de enseñanzas de régimen general de Educación Primaria (8), Secundaria (7) e inter-niveles (3), situados en Madrid ciudad (8) y otras localidades de la Comunidad (10). La mayoría pertenecen a la red pública (15), siendo uno privado y dos concertados. Como institución educativa, el Centro Regional de Innovación y Formación "Las Acacias", encargado de diseñar la gestión y actividades de formación del profesorado de la Comunidad de Madrid. Por otro lado, la Orquesta y Coro Nacionales de España, organismo que engloba los dos conjuntos estables de música sinfónico-coral del Ministerio de Educación, Cultura y Deporte.

A priori, el quehacer de los referidos centros educativos y el de la OCNE no tiene nada que ver. En cada uno de aquellos, centenares de chicos y chicas junto a decenas de docentes trabajan cada día desarrollando unas dinámicas rutinarias y pautadas, aunque no exentas de situaciones imprevisibles y estresantes. Al tiempo, conviven en un entramado de relaciones múltiples de gran complejidad. También hay unas diferencias acusadas entre la mayoría de centros participantes, situados en entornos de nivel bajo o medio bajo, algunos con evidentes dificultades económicas y sociales que repercuten negativamente en las comunidades escolares, y una minoría de alto nivel, sin dificultades económicas y con otras demandas sociales. Salvo excepciones, la actividad musical está reducida a la mínima expresión, a causa de las regulaciones legales actuales y a su escasa valoración social (cf. Kubik, 2016; Rodríguez-Quiles, 2014a, b; 2016; 2017a, b).

Por su parte, la OCNE está integrada por un colectivo de la máxima cualificación musical, que constituye un micro universo de perfiles muy singulares, centrado en una intensa actividad que busca la excelencia artística. Con sede en un espacio único como es el Auditorio Nacional de Música, el tiempo de los casi dos centenares de músicos (más el equipo técnico) transcurre entre ensayos, estudio, investigación, conciertos, giras, grabaciones... Sin la presencia de jóvenes, y fuera del foco público del tiempo concreto del 'concierto', la atmósfera vital diaria en la que se desenvuelve cada intérprete se muestra externamente como una mezcla entre relajación y tensión artística.

La mayoría del alumnado, profesorado y familias de los centros participantes no ha estado previamente en un espacio como es el Auditorio Nacional ni tiene contacto habitual con ese ambiente musical. Desconocen todo sobre los códigos, rutinas, lenguaje, tiempos y procedimientos que articulan su funcionamiento, algo que también le sucede a la mayoría de intérpretes que participan en el programa respecto al ambiente educativo de un colegio o instituto de régimen general. Sin embargo, el equipo de educación artística del CRIF sí tiene un conocimiento directo y previo de estos dos micro universos. Durante unos meses, Adoptar un músico los pone en contacto, hace que se descubran el uno al otro, THE CONTENT OF THIS ARTICLE IS THE SOLE RESPONSIBILITY OF THE AUTHORS. THE REVISTA ELECTRÓNICA DE LEEME AND UNIVERSITAT DE VALĖNCIA ARE NOT LIABLE FOR ANY LEGAL ACTIONS THAT MAY ARISE INVOLVING THE ARTICLE'S CONTENT. REVISTA ELECTRÓNICA DE LEEME - LISTA ELECTRÓNICA EUROPEA DE MÚSICA EN LA EDUCACIÓN-HTTP://MUSICA.REDIRIS.ES.ISSN: 1575(CSIC), EBSCO PREMIER, ERIH+, GALE CENGAGE LEARNING, IN-RECS, IRESIE, LATINDEX, MIAR, OCLC WORLDCAT, RESH, REDIB, RILM CORE JOURNALS, SUDOC, ULRICHS, ZEITSCHRIFTDATENBANK, ESTA REVISTA ESTÁ PUBLICADA CON EL APOYO INSTITUCIONAL DE REDIRIS-CONSEJO SUPERIOR DE INVESTIGACIONES CIENTIFICAS Y ES DE ACCESO LIBRE. 
provocando la aparición de nuevos procesos creativos, sociales y educativos que generan impactos significativos entre quienes participan.

\subsection{Proceso de la investigación}

Antes del inicio de los trabajos, se acordó con los responsables de las instituciones implicadas, OCNE y CRIF, los términos y condiciones en los que se deberían desarrollar el proceso investigador, concretados en un documento protocolo de investigación en el que se contemplaban aspectos como: autor y contexto, tipo de investigación, personas implicadas, accesos a espacios y documentación, actividades a desarrollar, procedimientos, recursos, calendario e informe.

Durante el trabajo de campo, desarrollado entre octubre de 2013 y junio de 2014, se asistió a decenas de actividades relacionadas con el programa: talleres, ensayos, conciertos, sesiones de evaluación, que se han desarrollado en auditorios, centros de formación, centros educativos, etc. En dichas actividades presenciales se realizaron observaciones directas (en centros educativos, de formación y auditorios) y entrevistas semiestructuradas a informantes cualificados.

Como estrategia complementaria de obtención de datos, se aplicó un cuestionario, en formato online, al profesorado implicado. Además, se realizaron frecuentes reuniones y encuentros con responsables de las instituciones, OCNE y CRIF, numerosos contactos informales e investigación documental (tabla 2).

Tabla 2. Actividades presenciales realizadas.

\begin{tabular}{|c|l|c|c|c|}
\hline $\mathbf{N}^{\mathbf{0}}$ & \multicolumn{1}{|c|}{ Actividad } & CRIF & Auditorio Nacional & Centros educativos \\
\hline 28 & Observación de talleres & & $\mathrm{X}$ & $\mathrm{X}$ \\
\hline 5 & Observación de ensayo general & & $\mathrm{X}$ & \\
\hline 5 & Observación de concierto & $\mathrm{X}$ & & $\mathrm{X}$ \\
\hline 3 & Observación de evaluaciones & & $\mathrm{X}$ & \\
\hline 5 & Entrevistas a docentes & & $\mathrm{X}$ & \\
\hline 7 & Entrevistas a intérpretes & & $\mathrm{X}$ & $\mathrm{X}$ \\
\hline 4 & Entrevistas a coordinadores & $\mathrm{X}$ & $\mathrm{X}$ & \\
\hline 1 & Entrevista grupal a asesores & & \\
\hline & Investigación documental & & \\
\hline
\end{tabular}

\subsubsection{Observaciones}

La premisa inicial para la selección de las situaciones susceptibles de ser observadas fue la de incluir la mayoría de las actividades programadas durante el periodo de trabajo de campo (tabla 3). 
Tabla 3. Sesiones de observación (*: proyecto adicional)

\begin{tabular}{|c|c|c|}
\hline & Programadas & Realizadas \\
\hline Talleres en centros & 44 & 28 \\
\hline Ensayos generales & 4 & $4+1^{*}$ \\
\hline Conciertos & 4 & $4+1^{*}$ \\
\hline Evaluaciones & 4 & 3 \\
\hline
\end{tabular}

La estrategia utilizada durante las sesiones de observación fue la de asumir el rol de observador participante (Flick, 2004; Angrosino, 2012). Ya dentro del campo, cada sesión se documentó mediante un registro narrativo observacional (tabla 4). En principio, de carácter abierto para poder captar los sucesos sin limitación previa, aunque orientado en algunos aspectos básicos: descriptivos (I. Información) y focalizados (II. Desarrollo y III. Dinámica):

Tabla 4. Registro de observación

\begin{tabular}{|l|l|l|l|}
\hline I. Información & II. Desarrollo & III. Dinámica & IV. Valoración \\
\hline Fecha y lugar & Objetivos & Interacciones & Diseño \\
\hline Duración & Actividades & Comentarios & Implementación \\
\hline Participantes & Recursos & & Contenidos \\
\hline Contexto & Agrupamientos & & Cultura \\
\hline
\end{tabular}

Como material de apoyo se grabaron imágenes y audio para documentar en su contexto todas aquellas actividades y situaciones susceptibles de interés para la investigación. Posteriormente, se incorporó un instrumento de observación cerrado (en escala Likert) de los aspectos de valoración (IV), con una serie de indicadores clave relativos a las actividades de observación en las que había participación de alumnado (ver Anexo I). Los 60 indicadores incluidos se basaron en el Protocolo de observación recogido por Frechtling y Sharp (1997, p. 43-54).

\subsubsection{Entrevistas}

Para las 17 entrevistas realizadas, de carácter semi-estructurado (Kvale, 2011), se utilizó un guion base con una serie de temas a tratar mediante una batería inicial de preguntas (entre 18 y 23), variables según el desarrollo de cada entrevista y adaptadas en función del rol de la persona entrevistada: en la institución musical OCNE, coordinador e intérprete; en la institución educativa, asesor/a del CRIF o docente en centro educativo.

Como muestreo teórico, la relación de personas a entrevistar no estuvo predeterminada a priori, sino que se fue concretando durante el desarrollo de los trabajos con el propósito de maximizar las oportunidades de comparar (Strauss y Corbin, 2002) y en función de la circunstancia del colectivo de pertenencia, la relevancia de la información disponible, la accesibilidad, la disponibilidad y la no saturación. En la relación de intérpretes y docentes entrevistados se consideraron diversos factores adicionales en orden a su potencial interés respecto a la investigación: su presencia en proyectos diferentes, la diversidad de su ubicación y sus contextos, y las primeras observaciones practicadas. 
En el caso de la institución musical OCNE, las entrevistas se realizaron a los miembros del Área Socioeducativa y a 7 de los 19 intérpretes que participaron en los proyectos desarrollados. En el caso de la institución educativa CRIF, las entrevistas se realizaron al equipo de asesores del Departamento de Enseñanzas Artísticas y a 5 de los 20 docentes que participaron en los proyectos desarrollados.

\subsubsection{Cuestionario}

El cuestionario dirigido al profesorado implicado, con acceso online para su cumplimentación una vez finalizado el desarrollo de los proyectos, se estructuró en seis bloques: Contexto, Organización, Agentes, Currículo y Prácticas, Resultados e Impacto, y Valoración. Incluía un total de 76 preguntas, de las cuales, 66 eran de respuesta cerrada (8, de contexto; 58, de contenido, en escala Likert, de 1 a 5) y 10 lo eran de respuesta abierta textual (Ver Anexo II).

Antes de aplicar el cuestionario, se procedió a su necesaria validación por 10 doctores, docentes universitarios, expertos en el campo, atendiendo a aspectos tales como: Pertinencia, Suficiencia, Claridad, Formato, Extensión, Presentación, Contenidos, Bloques, Orden y Redacción (ver Anexo III).

Recordamos que los objetivos de la investigación no son referidos a actividades puntuales sino a un programa estable en el tiempo. Es por ello que, si bien los datos de campo se recogen en el periodo ya señalado, también interesa incorporar datos que afectan a periodos anteriores del programa o, como en el caso de los cuestionarios, pueden ser aportados por informantes que hubieran participado en periodos anteriores al curso 2013-2014.

Al profesorado se le solicitaba su colaboración en función de su participación, que respondía a una premisa organizativa específica para el programa: convocatoria anual de un número de plazas limitado, teniendo prioridad los centros que no hubiesen participado en ediciones de cursos anteriores, por lo que la mayoría no puede participar más de un curso. Así, se remitió correo electrónico al profesorado de música que pudo ser identificado como participante en cualquiera de las convocatorias entre 2004 y 2014; en total, 129 docentes. Durante el periodo en que estuvo activado el cuestionario (40 días), se recibieron un total de 36 cuestionarios válidos, lo que supone una tasa de respuesta del 27,9\%.

\section{Análisis de resultados}

Para el análisis de la diversa tipología de información y datos obtenidos se procedió a su integración en un proceso de categorización y codificación, utilizando SPSS como programa de tratamiento informático para datos cuantitativos y NVivo para datos cualitativos, atendiendo a los dos tipos que recoge Gibbs (2012): por conceptos y por datos.

Como referencia inicial, y desde una lógica deductiva, se disponía de un sistema previo de categorías derivado de los temas incluidos en el propio proyecto de investigación y de la estructura del cuestionario elaborado. A partir del tratamiento de la información y los datos obtenidos, se trabajó de 
forma inductiva en una codificación abierta, identificando de forma significativa las categorías emergentes, práctica que conecta con el procedimiento de análisis desarrollado por Strauss y Corbin (2002, p.110).

Relacionando entre sí las categorías obtenidas en el paso anterior, se elaboró una primera estructura tentativa de categorías que, en relación con los objetivos de la investigación, permitiera establecer el sentido global de la misma, los temas centrales de análisis y los nodos ramificados asociados. De forma complementaria, se contrastó con la familia de códigos propuesta por Bogdan y Biklen (2007, p.185). Así, en la figura 1 se puede ver conceptualmente la estructura para el análisis relativa al programa en sí y a las personas que interviene en el mismo, desde varias direcciones: los procesos clave que desarrollan sus protagonistas, los planteamientos y premisas de cada colectivo, y las dinámicas que se establecen.

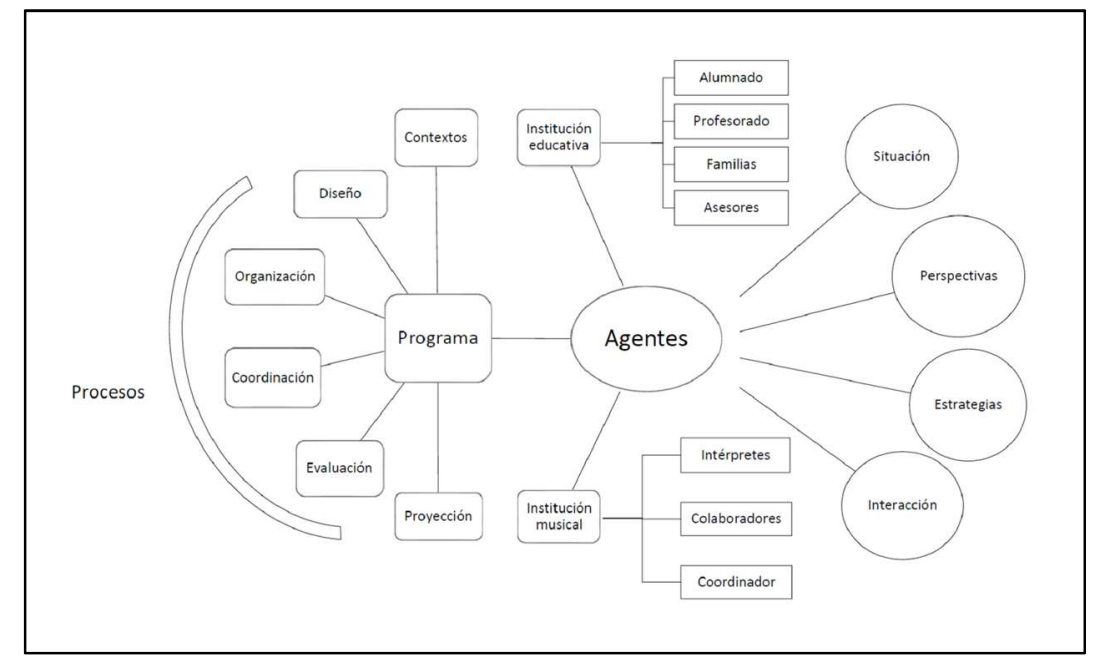

Fig. 1. Estructura de análisis 1 
En relación a los diferentes impactos que se derivan del programa, la figura 2 ofrece un esquema general.

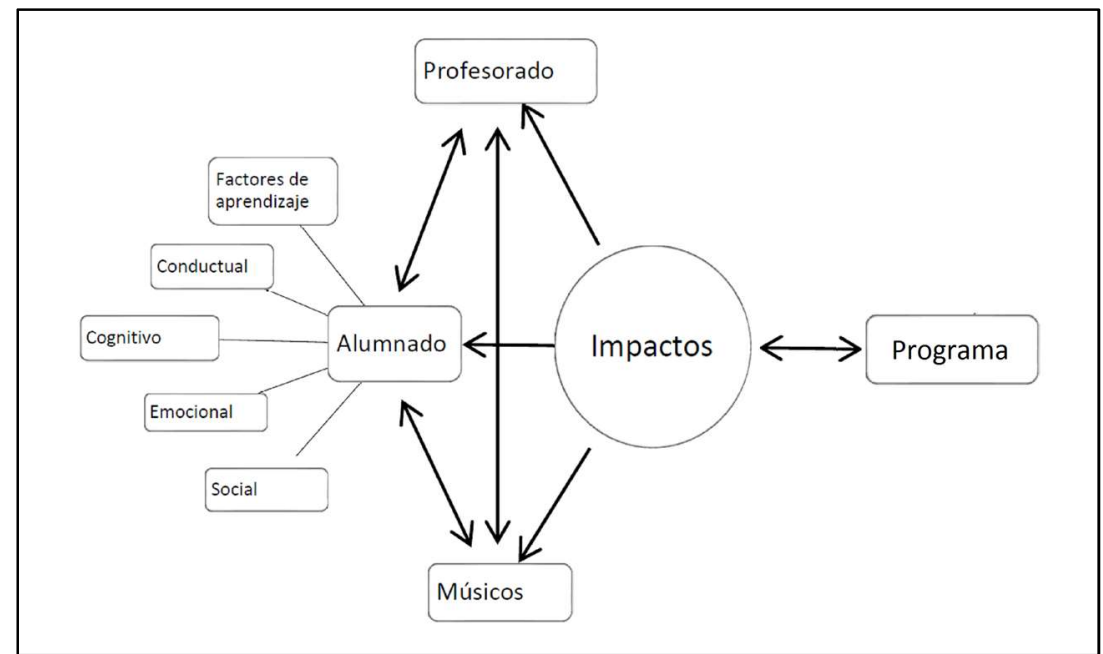

Fig. 2. Estructura de análisis 2

En cuanto a las subcategorías de impacto asociadas al alumnado, se han evidenciado incidencias en aspectos de diferentes factores: aprendizaje, cognitivo, conductual, emocional y social. En la figura 3 se puede observar la intensidad de los más significativos en función de su frecuencia en el análisis, es decir, el número de veces que se puede apreciar cada uno de los aspectos en el conjunto de datos analizados.

Un análisis más profundo del material cualitativo, que excedería los límites de esta publicación, nos podría ilustrar con más precisión respecto a cuáles son los factores, dentro de los señalados, que son realmente más relevantes en la práctica de los procesos educativos presentes en el caso.

Respecto al profesorado, los datos obtenidos del contexto muestran un perfil mayoritariamente femenino, con edad media de 40.5 años (desviación estándar 7.3), que trabaja en un centro público de entorno medio-medio bajo, y con una experiencia media como docente de música de 16.9 años (desviación estándar 7.6). Aprecia un alto grado de consecución de los objetivos del programa y lo valora como claramente innovador, ejemplo de buena práctica educativa y con fuerte incidencia, tanto en el currículo como en el proceso educativo. Lo percibe con un impacto global muy positivo y significativo, mayor al de otros programas y considera que tiene un alto nivel de repercusión en el centro educativo, las familias y el entorno próximo. También se constatan efectos sensibles tanto en relación con su propia actitud, motivación e implicación dentro del contexto laboral como en el desarrollo de su práctica profesional.

La figura 4 recoge los resultados de las respuestas del profesorado a los ítems de respuesta cerrada en el cuestionario sobre aspectos de currículo y prácticas. 


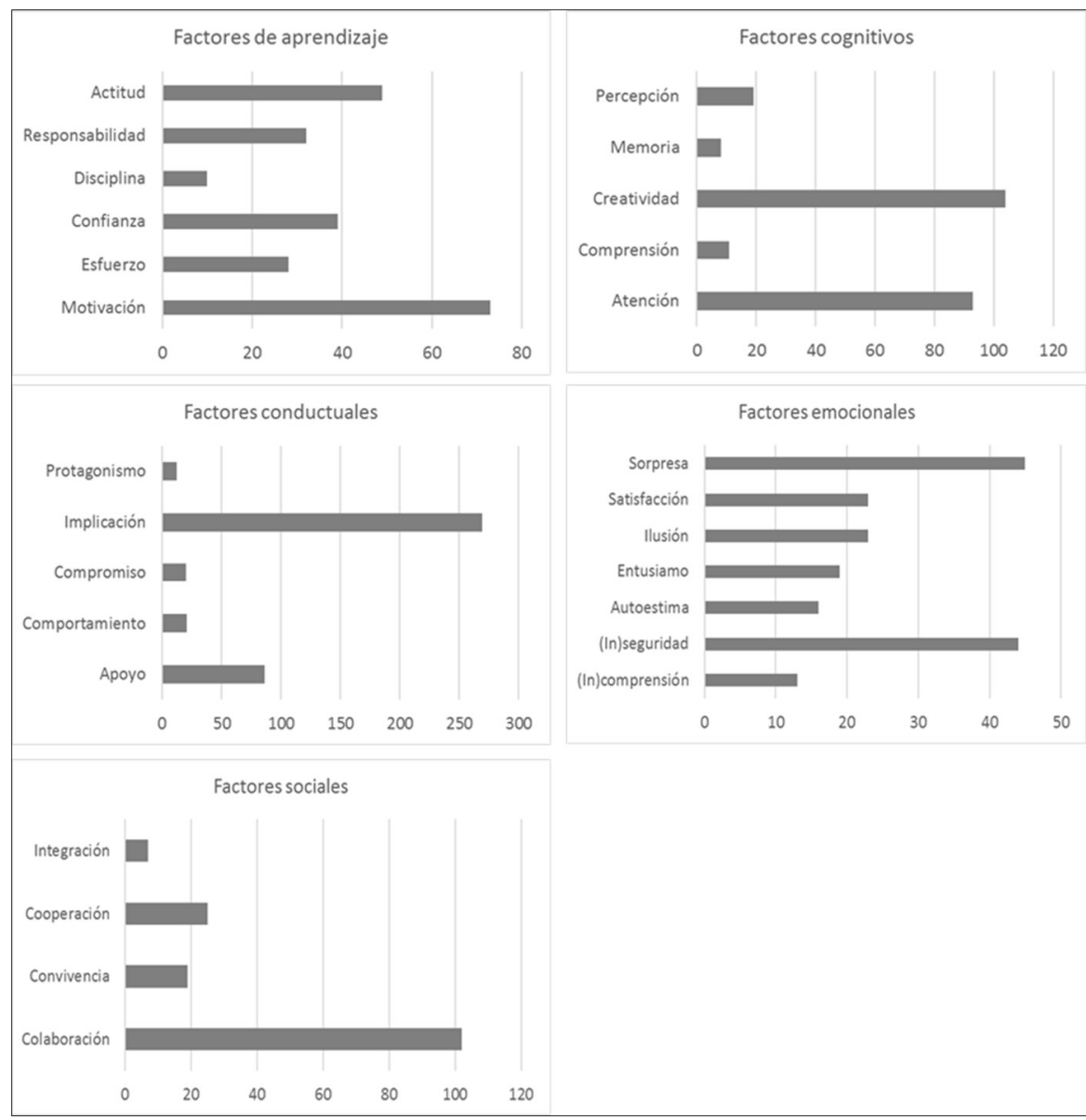

Fig.3. Factores de impacto

Desarrolla de contenidos del currículo Artística / Música Adquisición de nuevas habilidades y conocimientos $\mathrm{A} / \mathrm{M}$ Desarrolla habilidades y conocimientos otras áreas Desarrolla de las competencias básicas en el alumnado La metodología utilizada fue la adecuada para el programa Fomenta la creatividad como motor de aprendizaje Cambios en la forma de pensar y/o las prácticas del alumnado Influencia del programa sobre el alumnado en motivación Influencia sobre el alumnado en sensibilización musical Influencia sobre el alumnado en proyección hacia intereses musicales Influencia sobre el alumnado en crecimiento y autoestima personal Influencia sobre el alumnado en habilidades sociales y comunicativas Influencia sobre el alumnado en cooperación e integración social

El programa impulsó el desarrollo de mis habilidades creativas El programa mejoró mi confianza y motivación como docente El programa motivó un cambio en mi forma de trabajar

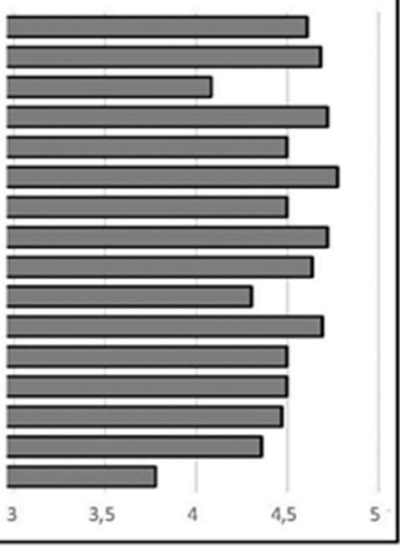

Fig. 4. Currículo y prácticas. 


\section{Discusión y conclusiones}

La visualización de programas educativos como el que constituyen el objeto de la presente investigación es percibida como puntual en el tiempo, circunscrita a eventos especiales, de carácter educativo no formal y periféricos al trabajo educativo formal desarrollado regularmente en las aulas. Con alguna excepción puntual, los datos recogidos durante el trabajo de campo lo evidencian, tanto desde dentro como desde fuera de la comunidad educativa. En el interior de la propia comunidad educativa, con mayor o menor intensidad en función de la proximidad o lejanía a la propia actividad. Es decir, mayor en las familias, en educación secundaria que en primaria, y en la propia administración educativa.

Pero lo cierto es que podríamos hacer una analogía con la estructura de un iceberg, en la que se aprecia un espectáculo fascinante por encima de la línea de flotación pero que necesita de una ingente masa, oculta a nuestra mirada, que sustente esa mínima porción del conjunto. En esa zona invisible es donde se sitúa el trabajo que el profesorado de música ha desarrollado junto al alumnado a lo largo de todo un curso escolar. Un trabajo que se ha integrado en el plan de estudios, en diferente grado y amplitud, y ha constituido la base de sus prácticas curriculares de carácter regular. Por tanto, con carácter central en el contexto de la educación formal.

La investigación ha permitido identificar y analizar algunos de los efectos que produce el programa Adoptar un músico en sus participantes. Desde luego que la experiencia no ha estado exenta de dificultades y de riesgos. Quizás, el más relevante sea la incógnita de aventurarse a salir de lo cómodo y rutinario, asociado a un concepto educativo estático, para explorar nuevos territorios a través de un modelo de aprendizaje diferente.

En primer lugar, el reconocimiento y valoración del carácter creativo del programa. El trabajo ha partido de la manipulación de elementos y células de una obra original del repertorio clásico para reelaborar una nueva obra musical. Es un proceso complejo en el que se trabaja de forma fragmentaria hasta que se van encontrando conexiones y se consigue ensamblar todo el material en la última fase del concierto. Es interesante destacar el carácter colectivo, donde las aportaciones individuales (las de un docente, un alumno, el compositor, o la improvisación que ha hecho el músico) quedan integradas e indiferenciadas dentro de un conjunto. Es una actividad que empieza siendo colaborativa y avanza en sentido cooperativo, mezclando distintos niveles y expectativas dentro de un espacio que invita a que se experimente sin miedo al error. También es un proceso creativo entendido como generador de un proyecto común entre los distintos centros educativos y la OCNE. Escuchar a "los otros" y crear "con otros" para un objetivo compartido cuya consecución se visualiza en el concierto final ante el público. Esta forma de entender la música como algo vivo, práctico, donde se aprende haciendo, ha sido lo más difícil y comprometido para quienes han participado en el programa, pero también lo más estimulante y enriquecedor. 
Los datos indican efectos relevantes del programa sobre factores emocionales, claves por su incidencia en el aprendizaje a través de la atención, la motivación y la conducta. También se han identificado algunos factores que intervienen, concurrentes y diferenciadores respecto a otras actividades: la participación activa en todo el proceso; la práctica musical, "hacer música", instrumental o vocal, dándoles la oportunidad de componer su propia música; la relación con músicos profesionales; la intervención como intérpretes en conciertos públicos, en un escenario singular. Un conjunto que, lejos de adjudicarles el rol de sujetos pasivos, los convierte en protagonistas de sus propias experiencias musicales, dentro y fuera del aula.

La experiencia acumulada desde el inicio del programa permite acreditar cómo la colaboración entre docentes de música, tutores y otro profesorado en torno a un programa como Adoptar un músico puede generar una incidencia positiva tanto en la educación musical como en otras áreas del currículo, así como en el bienestar general de la comunidad escolar. En este sentido, se puede citar la conexión de esta investigación y sus resultados con otras investigaciones en las que específicamente también se producía el encuentro entre institución escolar e institución educativa, orquesta y escuela, dentro de modelos participativos similares: Abeles (2004), DeNardo (1997), Henry et al. (2011).

La investigación también ha permitido descubrir el potencial educativo que tiene la combinación de músicos y diferentes personas expertas, ajenas al ámbito escolar, junto al profesorado de los centros educativos, especialmente de música y al propio alumnado. De alguna forma, unos y otros han de transgredir sus propias barreras y descubrir "al otro" en contextos no previsibles, situándose en un proceso adaptativo mutuo que incluye una "negociación" implícita.

En este sentido, se debe valorar la importancia de la función de mediación y animación de los integrantes del área socioeducativa de la OCNE y de asesores del CRIF. Además de su rol de dinamizadores de la actividad respecto a los objetivos planteados en el programa, desempeñan el de "traductores" que buscan el entendimiento y equilibrio entre dos ecosistemas diferentes, el cultural de la institución musical, más interesada objetivamente en el producto artístico resultante, y el educativo del centro escolar, que prioriza el valor de los procesos de enseñanza y aprendizaje.

Cabe destacar también la fortaleza interna que ha mostrado del programa que, e sí mismo, ha generado progresivamente un micro mundo de experiencias, retos y complicidades compartidas. Esto también se puede apreciar como una de sus debilidades en algunos casos por una tendencia al encapsulamiento respecto al contexto. Otro aspecto frágil es el alcance de su dimensión cuantitativa, ya que afecta, relativamente, a un porcentaje muy pequeño de la población escolar. Así mismo, se han detectado necesidades de mejora respecto a un mayor apoyo y colaboración institucional, programación de sesiones, comunicación y continuidad de la experiencia, entre otros aspectos.

Una dificultad subyacente en los procesos que se desarrollan en el programa es cómo hacer compatibles en la práctica los roles, tiempos, sistemas y valores de dos ámbitos institucionales complejos. Desde una mirada más amplia, la experiencia demuestra que la 
intersección entre ambos, desde la música, puede generar un poder multiplicador en la mejora de la educación y en la vida de las personas. Para ello, se han de favorecer los factores que debiliten las resistencias al cambio, potencien una vivencia significativa en sus protagonistas y alienten su capacidad de empoderamiento.

Se percibe como necesario que se pudieran acometer otras líneas de investigación complementarias que, sin carácter exhaustivo, se apuntan:

Una, en el particular contexto de uno o varios centros educativos, constituido en estudio de caso, y orientado a indagar en dos vertientes. Por un lado, cuáles son los resultados e impactos locales derivados de su participación en un programa educativo de los casos seleccionados. Por otro, estudios en profundidad de variables como las aquí recogidas en currículo y prácticas, cuando cada una de ellas se convierte en hipótesis de la investigación.

Otra, el diseño de una investigación que se ocupe de un mismo grupo de alumnos participante en uno de los programas, en dos fases. La primera, a lo largo del curso escolar, indagando dentro de su propio contexto, tanto en prácticas y aprendizajes como en otros impactos asociados. En esta dirección, cabe recordar el trabajo de Kushner (1991). La segunda, con carácter longitudinal, a lo largo de un periodo más dilatado, que permita determinar las dinámicas persistentes asociadas a su participación en estos programas. En ambos casos, con la posibilidad de incorporar un grupo de contraste con alumnado no participante.

Desde la perspectiva de los agentes educativos, la participación de músicos de máximo nivel artístico en el programa les ha posibilitado experiencias muy alejadas de su rol tradicional en la orquesta, ofreciéndoles la apertura a otras líneas de desarrollo profesional desde una óptica educativa que puede resultar problemática en algunos casos y gratificante en otros. Explorar cómo se desarrolla y qué efectos tiene esa participación también resultaría objeto de una posible investigación específica.

Finalmente, se requiere acometer investigaciones evaluativas en profundidad, con equipos multidisciplinares, de otros programas educativos que se están desarrollando en las Instituciones Musicales. Si bien es necesario atender a programas similares a los aquí tratados, también sería de interés que la selección de casos pudiera manejar criterios no coincidentes con los utilizados en este trabajo, a fin de ampliar el conocimiento de este apasionante campo. 


\section{Referencias}

Abeles, H. (2004). The effect of three orchestra school partnerships on students' interest in instrumental music instruction. Journal of Research in Music Education, 53 (3), 248-263.

Angrosino, M. (2012). Etnografia y observación participante en investigación Cualitativa. Madrid: Morata.

ABO (2014). The State of Britains Orchestras in 2013. Association of British Orchestras. Recuperado de http://www.abo.org.uk/media/32152/ABO-The-State-of-Britains-Orchestras.pdf

AFO (2014). Les publics de l'orchestre. Association Française des Orchestres. Recuperado de http://www.france-orchestres.com/colloque/wp-content/uploads/sites/3/2015/10/Synthèse-

Enquête-sur-les-publics-de-lorchestre-2013-14.pdf

Blázquez, S. y Bidegain, A. (2016). Gestión y recursos para la implantación de proyectos educativos en auditorios: el caso del Auditori Educa. (Tesis de Máster). Madrid: Instituto Complutense de Ciencias Musicales-Universidad Computense de Madrid.

Bogdan, R. y Biklen, S. (2007). Qualitative research for education: An introduction to theories and methods. New York: Allyn \& Bacon.

Bresler, L. (2006). Paradigmas cualitativos en investigación musical. En M. Díaz (Coord.), Introducción a la investigación en educación musical (pp. 60-81). Madrid: Enclave Creativa.

Burke, R. y Onwuegbuzie, A. (2004). Mixed Methods Research: A Research Paradigm Whose Time Has Come. Educational Researcher, 33 (7), 14-26.

Burke, R., Onwuegbuzie, A., y Turner, L. (2007). Toward a Definition of Mixed Methods Research. Journal of Mixed Methods Research, 1 (2), 112-133.

Cabedo, A. y Díaz, M. (2016). Educación musical y acción comunitaria: una visión de conjunto. Eufonía, 66, 4-6.

Cañas, M. (2008). Los conciertos didácticos en la educación musical. Granada: Centro de Documentación Musical de Andalucía.

Creswell, J.W. (2007). Qualitative inquiry and research design: Choosing among five approaches. London: Sage Publications.

Creswell, J.W. (2008). Mixed Methods Research. En L. Given (Ed.), The SAGE Encyclopedia of Qualitative Research Methods (pp. 526-529). London: Sage Publications.

Creswell, J., y Planco-Clark, V. (2011). Designing and conducting mixed methods research. London: Sage Publications.

THE CONTENT OF THIS ARTICLE IS THE SOLE RESPONSIBILITY OF THE AUTHORS. THE REVISTA ELECTRÓNICA DE LEEME AND UNIVERSITAT DE VALĖNCIA ARE NOT LIABLE FOR ANY LEGAL ACTIONS THAT MAY ARISE INVOLVING THE ARTICLE'S CONTENT. REVISTA ELECTRONICA DE LEEME : HTTPS.// OJS.UV.ES/INDEX.PHP/LEEME HTTP://MUSICA.REDIRIS.ES ISSN: 15759563 EDITORES: UNIVERSIDAD DE VALENCIA Y JESÚS TEJADA GIMENEZ. VISIBILIDAD DE ESTA REVISTA: EBSCO, CINDOC (CESIC), CITEFACTOR, COPAC, DIALNET, DICE (CSIC), DOAJ, E-REVISTAS (CSIC), EBSCO PREMIER, ERIH+, GALE CENGAGE LEARNING, IN-RECS, IRESIE, LATINDEX, MIAR, OCLC WORLDCAT, REDIB, RESH, RILM CORE JOURNALS, SUDOC, ULRICHS,

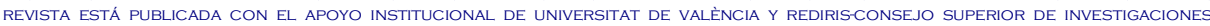
CIENTIFICAS Y ES DEACCESO UBRE. 
DeNardo, G. (1997). An Assessment of Student Learning in the Milwaukee Symphony Orchestra's ACE Partnership: 1991-2000. Bulletin of the Council for Research in Music Education, 148, 37-47.

Díaz, M. (Coord.) (2006). Introducción a la investigación en educación musical. Madrid: Enclave Creativa.

Elliott, J. y Kushner, S. (2007). The need for a manifesto for educational programme evaluation. Cambridge Journal of Education, 37 (3), 321-336.

Ferrada, V. (2008). Evaluación del proyecto Ensayos de OBC del Auditorio de Barcelona para alumnos de ESO (Trabajo de investigación de doctorado). Universidad Autónoma de Barcelona. Recuperado de http://www.recercat.cat/bitstream/handle/2072/13272/TESINA\%20FINAL$\underline{1 \% 20 V A N I A . p d f ? \text { sequence }=1}$

Flick, U. (2004). Introducción a la investigación cualitativa. Madrid: Morata

Flinders, D. y Richardson, C. (2002). Contemporary Issues in Qualitative Research and Music Education. En L. Colwell y C. Richardson (Eds.), The New Handbook of Research on Music Teaching and Learning (pp. 1159-1175). New York: Oxford University Press.

Frechtling, J., y Sharp, L. (1997). User-Friendly Handbook for Mixed Method Evaluations. Arlington: The National Science Foundation.

Gibbs, G. (2012). El análisis de datos cualitativos en investigación cualitativa. Madrid: Morata.

Goetz, J. y LeCompte, M. (1988). Etnografía y diseño cualitativo en investigación educativa. Madrid: Morata.

Henry, M., Murray, K., Hogrebe, M. y Thayer, M. (2011). Positive Impact of a Symphony's Support of Elementary Music Education. Recuperado de http://nebula.wsimg.com/bcad582eaa3dfc189c021cebf5045194?AccessKeyId=E6A9F9AEAC8 5059B70C $1 \&$ disposition $=0 \&$ alloworigin $=1$

Higgins, L. (2013). Música Comunitaria: Facilitadores, Participantes y Amistad. Revista Internacional de Educación Musical, 1, 53-59.

Johnson, B. y Onwuegbuzie, A. (2004). Mixed Methods Research: A Research Paradigm Whose Time Has Come. Educational Researcher, 33, 7, 14-26.

Kelly, M. J. (2008). Evaluation Research. En L. Given (Ed.), The SAGE Encyclopedia of Qualitative Research Methods (pp. 303-306). London: Sage Publications.

Klassen, A.,Creswell, J., Plano, V., Clegg, K., y Meissner, H.(2012). Best practices in mixed methods for quality of life research. Quality of Life Research, 21, 3, 377-380.

Korn, M. (2000). Orchestras That Educate. Harmony, 10, 57-68.

THE CONTENT OF THIS ARTICLE IS THE SOLE RESPONSIBILITY OF THE AUTHORS. THE REVISTA ELECTRÓNICA DE LEEME AND UNIVERSITAT DE VALĖNCIA ARE NOT LIABLE FOR ANY LEGAL ACTIONS THAT MAY ARISE INVOLVING THE ARTICLE'S CONTENT. REVISTA ELECTRONICA DE LEEME- HTTPS:/ / OJS.UV.ES/INDEX.PHP/LEEME HTTP://MUSICA.REDIRIS.ES ISSN: 15759563 EDITORES: UNIVERSIDAD DE VALENCIA Y JESÚS TEJADA GIMÉNEZ. VISIBILIDAD DE ESTA REVISTA: EBSCO, CINDOC (CESIC), CITEFACTOR, COPAC, DIALNET, DICE (CSIC), DOAJ, E-REVISTAS (CSIC), EBSCO PREMIER, ERIH+, GALE CENGAGE LEARNING, IN-RECS, IRESIE, LATINDEX, MIAR, OCLC WORLDCAT, REDIB, RESH, RILM CORE JOURNALS, SUDOC, ULRICHS, SO REVISTA ESTÁ PUBLICADA CON EL APOYO INSTITUCIONAL DE UNIVERSITAT DE VALĖNCIA Y REDIRIS-CONSEJO SUPERIOR DE INVESTIGACIONES CIENTIFICAS Y ES DE ACCESO LIBRE. 
Kubik, S. (2016). L'éducation musicale en Espagne: et s'il n'y en avait plus du tout? Recuperado de https://www.francemusique.fr/actualite-musicale/1-education-musicale-en-espagne-et-s-il-ny-en-avait-plus-du-tout-238

Kushner, S. (1991). The Children's Music Book. London: Calouste Gulbenkian Foundation.

Kushner, S. (1998). Evaluación de programas de conciertos para escolares. El empleo de estudios de casos en la evaluación de la educación musical. En J. Palomares y G. Roldán (Eds.), Los conciertos didácticos (pp. 235-248). Granada. Centro de Documentación Musical de Andalucía.

Kushner, S. (2002). Personalizar la evaluación. Madrid: Morata

Kvale, S. (2011). Las entrevistas en investigación cualitativa. Madrid: Morata

Lacord (2011). Dossier de Premsa. Balanç de la temporada 2010-11 i presentació de la programació 2011-12. No publicado.

Lincoln, Y.S. y Guba, E.G. (1985). Naturalistic inquiry. Beverly Hills: Sage Publications.

Lukas, J.F., Santiago K. y Lizasoain, L. (2005). Vitoria-Gasteiz como Ciudad Educadora: Evaluación de los Programas del Departamento Municipal de Educación. Universidad del País Vasco.

http://www.sc.ehu.es/plwlumuj/ebaluazioa_pedagogia/praktikak/INFORME-vitoria.pdf

MacDonald, B. (1976). Evaluation and the control of education. En Tawney, D. (Ed.), Curriculum evaluation today: trends and implications (pp. 125-136). London: Macmillan.

Malagarriga, A. (2010). Que sea dificil dejar de escuchar. Barcelona: L'Auditori.

Moreiras, A. (2005). Evaluación de un concierto didáctico. En Eufonía, 33, 102-108.

Neuman, V. (2003). Los conciertos didácticos y la audición musical en el aula. La experiencia de la Orquesta Ciudad de Granada y su influencia en la acción docente (Tesis doctoral no publicada). Granada: Universidad de Granada.

Ortega, M. C. (2012). Adoptar un músico. En Díaz, M. (Coord.). Aulas del siglo XXI: retos educativos (pp. 341-371). Madrid: Instituto Nacional de Tecnologías Educativas y de Formación del Profesorado.

Ramírez, A. (2006). ¿Cómo es el público del Teatro Real de Madrid que asiste a la Ópera en Familia? (Tesis de Master). Madrid: Instituto Complutense de Ciencias Musicales-Universidad Complutense de Madrid.

ROCE. (2016). Los conciertos. Red de Organizadores de Conciertos Educativos. Recuperado de: https://rocemusica.org/roce/acciones/

THE CONTENT OF THIS ARTICLE IS THE SOLE RESPONSIBILITY OF THE AUTHORS. THE REVISTA ELECTRÓNICA DE LEEME AND UNIVERSITAT DE VALĖNCIA ARE NOT LIABLE FOR ANY LEGAL ACTIONS THAT MAY ARISE INVOLVING THE ARTICLE'S CONTENT. REVISTA ELECTRONICA DE LEEME * HTTPS://OJS.UV.ES/INDEX.PHP/LEEME HTTP://MUSICA.REDIRIS.ES ISSN: 15759563 EDITORES: UNIVERSIDAD DE VALENCIA Y JESÚS TEJADA GIMENEZ. VISIBILIDAD DE ESTA REVISTA: EBSCO, CINDOC (CESIC), CITEFACTOR, COPAC, DIALNET, DICE (CSIC), DOAJ, E-REVISTAS (CSIC), EBSCO PREMIER, ERIH+, GALE CENGAGE LEARNING, IN-RECS, IRESIE, LATINDEX, MIAR, OCLC WORLDCAT, REDIB, RESH, RILM CORE JOURNALS, SUDOC, ULRICHS, SE REVISTA ESTÁ PUBLICADA CON EL APOYO INSTITUCIONAL DE UNIVERSITAT DE VALĖNCIA Y REDIRIS-CONSEJO SUPERIOR DE INVESTIGACIONES CIENTIFICAS Y ES DEACCESO HIBRE. 
Rodríguez-Quiles, J. A. (2014a). Chateando en el aula a pequeños sorbos. Aspectos performativos en culturas digitales escolares. En J. F. Durán (Ed.), Aprendiendo en el nuevo espacio educativo superior (pp. 405-422). Madrid: ACCI.

Rodríguez-Quiles, J. A. (2014b). Minister Werts Werte. Oder das Ende der Musik im spanischen Schulsystem,en Musik Forum, 4, 14, 36-38.

Rodríguez-Quiles, J. A. (2016). Music Teacher Training: A precarious area within the Spanish university. British Journal of Music Education, 1-14. doi:10.1017/S026505171600036X

Rodríguez-Quiles, J. A. (2017a). Rethinking Music Education: Towards a Performative Turn. En J. A. Rodríguez-Quiles (Ed.). Internationale Perspektiven zur Musik(lehrer)ausbildung in Europa (pp. 21-40). Potsdam: UV.

Rodríguez-Quiles, J. A. (2017b). (En prensa). La música como rizoma. Bases para una educación musical performativa. Cultura y Educación.

Simons, H. (2011). El estudio de caso: Teoría y práctica. Madrid: Morata.

Stake, R. (1998). Investigación con estudio de casos. Madrid: Morata.

Stake, R. (2006). Evaluación comprensiva y evaluación basada en estándares. Barcelona: Graó.

Strauss, A. y Corbin, J. (2002). Bases de la investigación cualitativa: técnicas y procedimientos para desarrollar la teoría fundamentada. Medellín: Universidad de Antioquía.

Swol, W. (2005). Conciertos didácticos, ¿quién es su público y qué opina? Música y Educación, $63,47-61$.

Vinent, M. y Gustems, J. (2014). Conciertos didácticos en Barcelona: aportaciones a la educación musical. Música y Educación, 99, 4-49.

Yin, R. K. (2009). Case study research: design and methods. London: Sage Publications. 


\section{Anexo I}

\section{Registro de observación}

\section{Información general}

\begin{tabular}{|l|l|l|}
\hline \multicolumn{2}{|l|}{ Programa } & Lugar \\
\hline \multicolumn{2}{|l|}{ Observador } & Número total de asistentes \\
\hline Fecha de la observación & Horario de la observación & Duración de la observación \\
\hline $\begin{array}{l}\text { Número de sesión } \\
\text { Total de sesiones }\end{array}$ & $\begin{array}{l}\text { Tipos de agrupamiento utilizado: } \\
\text { Grupo completo Grupos pequeños } \quad \text { Individual }\end{array}$ \\
\hline \multirow{3}{*}{ Agentes educativos } & \\
\cline { 2 - 3 } & \\
\cline { 2 - 3 } Objetivos de la sesión & \\
\cline { 2 - 3 } & \\
\cline { 2 - 2 } & \\
\hline Recursos didácticos utilizados & \\
\hline
\end{tabular}

- Cada indicador se calificará en una escala entre 1 y 7.

- Para señalar la calificación adecuada en cada uno de los indicadores se debe elegir dentro de una escala de 1 (no, en absoluto) a 5 (completamente).

- Cuando se considere que no hay suficiente evidencia para señalar una calificación, se debe marcar 6 en la escala.

- Si alguno de los indicadores no fuera aplicable para la sesión observada, se debe marcar 7 en la escala.

- Si se considera necesario, se pueden incluir otros indicadores adicionales. 


\section{Diseño}

A. Indicadores

1. El diseño de la sesión se basa en una precisa planificación y organización

2. La sesión se estructura en base a un conocimiento previo por los participantes del proceso a seguir

3. Los objetivos de la sesión son claros y equilibrados

4. La metodología y actividades previstas son coherentes con los objetivos del programa

5. La metodología y actividades previstas consideran la experiencia, preparación previa y estilos de aprendizaje de los participantes

6. El diseño de la sesión impulsa un enfoque colaborativo para el aprendizaje

7. El diseño de la sesión incluye tareas, roles e interacciones coherentes con el programa

8. El diseño de la sesión permite aportar al profesorado recursos, estrategias y técnicas para el aula

9. El diseño de la sesión contempla un tiempo y un espacio adecuados para la reflexión e intercambio de puntos de vista

10

B. Valoración de Síntesis

El diseño de la sesión Si/No incorpora los elementos adecuados para el desarrollo del programa.

C. Evidencias a considerar para la valoración de síntesis: 


\section{Implementación}

A. Indicadores

1. La metodología y actividades son adecuadas y eficaces para el desarrollo de los objetivos previstos

2. La metodología y actividades son adecuadas y eficaces para la comprensión conceptual y de contenidos

3. El ritmo y la dinámica de la sesión favorecen el desarrollo de los objetivos previstos

4. El animador/mediador se adapta y responde a las necesidades e intereses de los participantes

5. La experiencia y/o conocimientos del animador/mediador (s) favorece la calidad de la sesión

6. El estilo y metodología del animador/mediador (s) estimula y favorece la calidad de la sesión

7. Se evidencia que la mayoría del grupo participa activamente en la sesión

8. La sesión incorpora estrategias de evaluación eficaces

9.

B. Valoración de Síntesis

La implementación de la sesión Es/No es un reflejo de buena práctica educativa.

C. Evidencias a considerar para la valoración de síntesis: 
LISTA ELECTRÓNICA EUROPEA

DE MÚSICA EN LA EDUCACIÓN

\section{Contenidos}

A. Indicadores

1. Los contenidos son adecuados para los objetivos de la sesión, preparación y perfil de los participantes

2. Los contenidos se presentan y desarrollan de forma clara y consistente

3. El animador/mediador demuestra un uso adecuado de los conceptos en su interacción con los participantes

4. Los contenidos se exponen de forma dinámica e interactiva

5. La profundidad y amplitud de los contenidos es la adecuada para los objetivos de la sesión y las necesidades de los participantes

6. El grado de comprensión conceptual es adecuado para los objetivos de la sesión y las necesidades de los participantes

7. La sesión incluye una atención explícita a cuestiones de aplicación en el aula

8. Se formulan conexiones con otras áreas/disciplinas y/o contextos

9.

B. Valoración de Síntesis

Los contenidos de la sesión Si/No incorporan las mejores prácticas para el desarrollo de los objetivos.

C. Evidencias a considerar para la valoración de síntesis: 


\section{Agentes educativos}

En esta sección se identifica a participante como el agente educativo activo en la sesión: animador, mediador, músico, docente... Se debe tener en cuenta toda la información disponible (observaciones de esta sesión, entrevistas relacionadas, conocimiento general del programa...) para una mejor valoración de cada uno de los aspectos señalados.

\section{A. Indicadores}

1. La capacidad de los participantes para implementar eficazmente el programa

2. La capacidad de los participantes para identificar y exponer con claridad las ideas del programa

3. La comprensión de los participantes respecto a las formas de aprendizaje de niños/as

4. La capacidad de los participantes para utilizar los materiales y recursos del programa

5. La sensibilidad de los participantes para integrar diferencias dentro del grupo respecto a su experiencia previa, preparación, género u origen étnico y/o cultural.

6. La proactividad de los participantes para detectar y resolver necesidades emergentes 7.

B. Valoración de Síntesis

El trabajo y el liderazgo de los agentes educativos en la sesión Es/No es adecuado y eficaz.

C. Evidencias a considerar para la valoración de síntesis: 
LISTA ELECTRÓNICA EUROPEA

DE MÚSICA EN LA EDUCACIÓN

\section{Cultura y valores}

A. Indicadores

1. Se fomenta y valora la participación activa de todos y todas

2. Hay un clima de respeto que favorece la implicación abierta de los participantes

3. Las interacciones reflejan relaciones de colaboración entre los participantes

4. Las interacciones reflejan relaciones de cooperación entre los participantes

5. Se impulsan condiciones favorables para la creatividad

6. Se aprovechan las oportunidades para reconocer y cambiar posibles estereotipos y prejuicios

7. Se valora y reconoce la iniciativa, la crítica constructiva y las nuevas ideas

8. Se favorece la conexión del programa con otras áreas artísticas y del currículum

9.

B. Valoración de Síntesis

La cultura y valores de la sesión dificultan/facilitan el desarrollo del programa y la integración de los participantes como miembros de una comunidad de aprendizaje.

C. Evidencias a considerar para la valoración de síntesis:

\section{Observaciones sobre la sesión:}




\section{Anexo II}

\section{Datos}

\section{Cuestionario al Profesorado}

1. Programa o programas en que participó

Scherzo de la $2^{\text {a }}$ Sinfonía. Beethoven (04-05)

El Pájaro de fuego / El Bolero (04-05)

Water Concerto (05-06)

Sinfonía No.9 (05-06)

El Amor Brujo (05-06)

El Cascanueces (06-07)

La Creación (06-07)

Historia de un soldado (06-07)

El mandarín maravilloso / Vers, l'Arc-en-ciel (07-08)

Cuadros de una exposición (07-08)

Historia del soldado / Rikadla (07-08)

West Side Story (08-09)

Carmina Burana (08-09)

Sheherezade (08-09)

Cuatro interludios marinos de Peter Grimes (09-10)

Cuatro danzas de Estancia / Tangazo (09-10)

Las Estaciones (09-10)

El Castillo de Barba Azul (09-10)

Estaciones Brujas (10-11)

2010 Odisea en el Espacio (10-11)

Músicas de Aquí y de Allá (10-11)

Alexander Nevsky (10-11)

El sombrero de tres picos (11-12)

El Mar (11-12)

El pájaro de fuego (11-12)

Dhapnis et Chloé (11-12)

Juego filarmónico (12-13)

Pulcinella (12-13)

Babi Yar (12-13)

El caballero de la rosa (12-13)

Una travesura musical (13-14)

Loop! (13-14)

Sueños de Oriente (13-14)

Dido Remix (13-14)

2. Nivel educativo

Educación Primaria

Educación Secundaria

3. Tipo de centro 
LISTA ELECTRÓNICA EUROPEA

DE MÚSICA EN LA EDUCACIÓN

\title{
Público
}

Privado concertado

Privado

4. Entorno socioeconómico y cultural donde considera que se ubica el centro

\author{
Bajo \\ Medio-Bajo \\ Medio \\ Medio-Alto \\ Alto
}

5. Edad

$$
\begin{aligned}
& \text { hasta } 30 \\
& \text { de } 31 \text { a } 40 \\
& \text { de } 41 \text { a } 50 \\
& \text { de } 51 \text { a } 60 \\
& \text { más de } 60
\end{aligned}
$$

6. Sexo
Mujer
Hombre

7. Años de experiencia como docente
menos de 5
de 5 a 10
de 11 a 15
de 16 a 20
de 21 a 25
de 26 a 30
más de 30

8. Años de experiencia como docente de música
menos de 5
de 5 a 10
de 11 a 15
de 16 a 20
de 21 a 25
de 26 a 30
más de 30 


\section{A. Organización}

1. Recibí información suficiente para el desarrollo del programa

2. El número de sesiones de trabajo fue suficiente para el desarrollo del programa

3. El calendario fue adecuado para el desarrollo del programa

4. En el desarrollo del programa se dispuso de recursos suficientes

5. El material disponible era adecuado para su uso educativo

6. La coordinación general del programa fue eficaz

7. Como docente, pude seguir sin dificultad la secuencia establecida para el desarrollo del programa

8. El alumnado pudo seguir sin dificultad la secuencia establecida para el desarrollo del programa

9. Nivel de implicación y/o apoyo del equipo directivo del centro al programa

10. Nivel de implicación y/o apoyo del profesorado del centro al programa

11. Nivel de implicación y/o apoyo de padres y madres al programa

12. Utilice el siguiente espacio si desea hacer algún comentario

\section{B. Agentes}

1. Como docente, mi comunicación e interacción con el asesor del CRIF fue (1, insuficiente -5 , óptima)

2. Como docente, mi comunicación e interacción con el colaborador OCNE fue (1, insuficiente -5 , óptima)

3. Como docente, mi comunicación e interacción con el músico fue (1, insuficiente 5, óptima)

4. La comunicación e interacción entre el colaborador OCNE y el alumnado fue (1, insuficiente -5 , óptima)

5. La comunicación e interacción entre músico y alumnado fue (1, insuficiente -5 , óptima) 
6. El alumnado entendió con facilidad las ideas y propuestas del colaborador OCNE

7. El alumnado entendió con facilidad las ideas y propuestas del músico

8. Asesor, colaborador y músico se adaptaron a las necesidades específicas de mi centro educativo

9. El ambiente durante las sesiones de trabajo fue abierto, receptivo y estimulante

10. El nivel de colaboración con los otros centros participantes fue (1, insuficiente -5 , óptimo)

11. Utilice el siguiente espacio si desea hacer algún comentario

\section{Currículo y prácticas}

1. El programa propició el desarrollo de los contenidos del currículo de Ed. Artística/Música

2. El programa facilitó la adquisición de nuevas habilidades y conocimientos en Ed. Artística/Música

3. El programa ayudó a desarrollar habilidades y conocimientos en otras áreas del currículo escolar

4. El programa contribuyó al desarrollo de las competencias básicas en el alumnado

5. La metodología utilizada fue la adecuada para el desarrollo del programa

6. El programa potenció la creatividad como motor de aprendizaje del alumnado

7. El programa generó cambios en la forma de pensar y/o las prácticas del alumnado

8. Mi percepción respecto a la influencia del programa en el alumnado
a. Motivación
b. Sensibilización musical
c. Proyección hacia intereses musicales
d. Crecimiento y autoestima personal
e. Habilidades sociales y comunicativas
f. Cooperación e integración social

9. El programa impulsó el desarrollo de mis habilidades creativas

10. El programa mejoró mi confianza y motivación hacia el trabajo docente

11. El programa motivó un cambio en mi forma de trabajar: práctica, metodología, organización del aula... 
12. Utilice el siguiente espacio si desea hacer algún comentario

\section{Resultados e Impacto}

1. Grado de consecución de objetivos del programa

a) Conseguir una escucha musical más activa y participativa, convirtiendo en creadores e intérpretes al profesorado y alumnado.

b) Acercar al alumnado a los elementos y estructuras de la música, haciéndoles partícipes en las fases de creación e interpretación.

c) Estimular la creatividad artística y el espíritu crítico de los participantes.

d) Entender y disfrutar la riqueza del patrimonio musical

e) Promover relaciones entre la OCNE, profesorado y alumnado, desmitificando la música sinfónico-coral y a sus protagonistas.

2. Nivel de repercusión en el centro educativo

3. Nivel de repercusión en las familias

4. Nivel de repercusión en el proceso educativo

5. Nivel de repercusión en el entorno próximo

6. El programa es innovador y relevante para el desarrollo de la función educativa

7. Considero el programa como ejemplo de buena práctica educativa

8. Valore el impacto comparativo que estima en relación con otras iniciativas y programas en que haya participado $(1$, mucho menor -5 , mucho mayor $)$

9. El impacto global del programa es (1, negativo y nada significativo - 5, muy positivo y significativo)

10. Utilice el siguiente espacio si desea hacer algún comentario

\section{E. Valoración}


1. Asesor CRIF: metodología, colaboración, aportaciones..

2. Colaborador OCNE: metodología, colaboración, aportaciones...

3. Músico: metodología, colaboración, aportaciones...

4. Valoración de las fases del programa
a. Formación
b. Talleres en el aula
c. Asistencia al ensayo OCNE
d. Ensayos generales
e. Concierto

5. ¿Cuáles considera que son los aspectos más valiosos del programa?

6. ¿Qué dificultades tuvo para el desarrollo del programa?

7. ¿Qué aspectos del programa considera que se pueden mejorar?

8. ¿Qué nuevos elementos debería incorporar el programa?

9. Valoración general de la experiencia en el programa

10. Utilice el siguiente espacio si desea hacer algún comentario 


\section{Anexo III}

\section{Cuestionario al Profesorado}

Validación de expertos

Valoración del conjunto de ítems que conforman los aspectos que se incluyen en el cuestionario, según el grado de acuerdo con los criterios indicados ( 1 = inadecuado / 5 = muy adecuado).

\begin{tabular}{|l|c|c|c|c|c|}
\hline ASPECTOS & ITEMS & Pertinencia & Suficiencia & Claridad & Formato \\
\hline Datos & $\mathbf{1}-\mathbf{8}$ & 4,55 & 4,33 & 4,44 & 4,00 \\
\hline Organización & A.1 - A.12 & 4,50 & 4,50 & 4,25 & 3,87 \\
\hline Agentes & B.1 - B.11 & 4,50 & 4,37 & 3,62 & 3,87 \\
\hline Currículo y prácticas & C.1 - C.12 & 4,50 & 4,25 & 4,25 & 3,50 \\
\hline Resultados e impacto & D.1 - D.10 & 4,37 & 4,62 & 3,62 & 3,75 \\
\hline Valoración & E.1 - E.10 & 4,62 & 4,50 & 4,00 & \\
\hline
\end{tabular}

\begin{tabular}{|l|c|}
\hline Extensión del cuestionario en su conjunto & 4,25 \\
\hline Presentación del cuestionario en su conjunto & 4,12 \\
\hline
\end{tabular}

